\title{
Correction to: Analysis of nocturnal desaturation waveforms using algorithms in patients with idiopathic pulmonary fibrosis
}

\author{
Yuichiro Yasuda ${ }^{1} \cdot$ Tatsuya Nagano $^{1} \cdot$ Shintaro Izumi $^{2} \cdot$ Mina Yasuda $^{3} \cdot$ Kosuke Tsuruno $^{3} \cdot$ Kazunori Tobino $^{3}$. \\ Kyosuke Nakata ${ }^{1,4} \cdot$ Kayoko Okamura $^{5} \cdot$ Teruaki Nishiuma $^{6} \cdot$ Kiyonobu Takatsuki $^{7}$. Yasuhiro Funada ${ }^{8}$. \\ Hisashi Ohnishi ${ }^{5}$. Masatsugu Yamamoto ${ }^{1}$. Yoshihiro Nishimura ${ }^{1} \cdot$ Kazuyuki Kobayashi $^{1}$ \\ Published online: 12 January 2022 \\ (c) Springer Nature Switzerland AG 2021
}

\section{Correction to: Sleep and Breathing}

https://doi.org/10.1007/s11325-021-02456-3

Figure 4 of the published article contained an error. The correct figure is shown below.

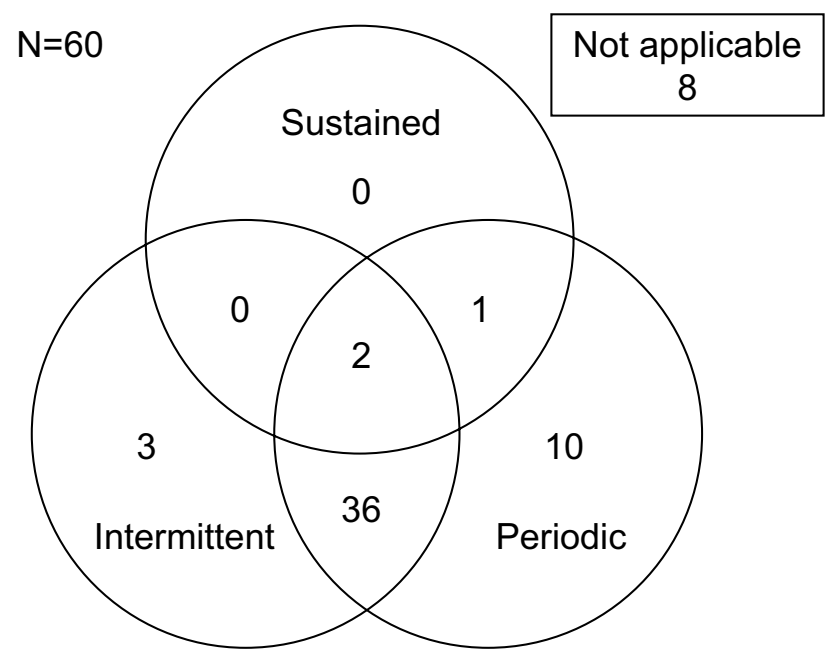

Fig. 4 Venn diagram of each waveform in patients with idiopathic pulmonary fibrosis

The original article can be found online at https://doi.org/10.1007/ s11325-021-02456-3

Tatsuya Nagano

tnagano@med.kobe-u.ac.jp

1 Department of Internal Medicine, Division of Respiratory Medicine, Kobe University Graduate School of Medicine, 7-5-1 Kusunoki-cho, Chuo-ku, Kobe, Hyogo 650-0017, Japan

2 Graduate School of System Informatics, Kobe University, 1-1-Rokkodai-cho, Nada-ku, Kobe, Hyogo 657-8501, Japan

3 Department of Respiratory Medicine, Iizuka Hospital, 3-83 Yoshiomachi, Iizuka, Fukuoka 820-0018, Japan
Publisher's note Springer Nature remains neutral with regard to jurisdictional claims in published maps and institutional affiliations.
4 Department of Respiratory Medicine, Kasai City Hospital, 1-13 Yoko, Hojo-cho, Kasai, Hyogo 675-2311, Japan

5 Department of Respiratory Medicine, Akashi Medical Center, 743-33 Yagi, Okubo-cho, Akashi, Hyogo 674-0063, Japan

6 Department of Respiratory Medicine, Kakogawa Central City Hospital, 439 Honmachi, Kakogawa-cho, Kakogawa, Hyogo 675-8611, Japan

7 Department of Respiratory Medicine, Kitaharima Medical Center, 926-250 Ichiba-cho, Ono, Hyogo 675-1392, Japan

8 Department of Respiratory Disease, Takatsuki General Hospital, 1-3-13 Kosobe-cho, Takatsuki, Osaka 569-1192, Japan 